\title{
Percepción del riesgo asociado al volcán Cotopaxi y vulnerabilidad en el Valle de Los Chillos (Ecuador)
}

Perception du risque lié au volcan Cotopaxi et vulnérabilité dans la vallée de Los Chillos (Équateur)

Cotopaxi volcano risk perception and vulnerability in Los Chillos Valley

(Ecuador)

Diana Salazar y Robert D’Ercole

\section{OpenEdition}

Journals

Edición electrónica

URL: http://journals.openedition.org/bifea/2522

DOI: 10.4000/bifea.2522

ISSN: 2076-5827

Editor

Institut Français d'Études Andines

Edición impresa

Fecha de publicación: 1 diciembre 2009

Paginación: 849-871

ISSN: 0303-7495

Referencia electrónica

Diana Salazar y Robert D'Ercole, « Percepción del riesgo asociado al volcán Cotopaxi y vulnerabilidad en el Valle de Los Chillos (Ecuador) », Bulletin de I'Institut français d'études andines [En línea], 38 (3) | 2009, Publicado el 01 junio 2010, consultado el 17 noviembre 2020. URL : http:// journals.openedition.org/bifea/2522 ; DOI : https://doi.org/10.4000/bifea.2522

Les contenus du Bulletin de l'Institut français d'études andines sont mis à disposition selon les termes de la licence Creative Commons Attribution - Pas d'Utilisation Commerciale - Pas de Modification 4.0 International. 


\title{
Percepción del riesgo asociado al volcán Cotopaxi y vulnerabilidad en el Valle de Los Chillos (Ecuador)
}

\author{
Diana Salazar* \\ Robert D'Ercole**
}

\section{Resumen}

En 2007 se realizó una encuesta sobre la percepción del riesgo asociado al volcán Cotopaxi en el Valle de Los Chillos, situado cerca de Quito. De ella se desprenden la percepción de la amenaza volcánica y sus características, la representación de los espacios más expuestos a los fenómenos volcánicos y la percepción del riesgo en el lugar de residencia. Los resultados de la encuesta evidencian una conciencia clara de la existencia del riesgo volcánico, pero también distorsiones significativas entre el punto de vista de los científicos y el de la población con respecto a las zonas más expuestas. Dadas las incertidumbres que caracterizan al riesgo volcánico, este tipo de investigación es muy útil en términos de acción preventiva y de reducción de la vulnerabilidad de la población. Su propósito no es obtener la adhesión de un máximo de población a un punto de vista único, considerado como objetivo (el de los científicos), sino acercar de manera constructiva los puntos de vista de los diferentes actores.

Palabras clave: percepción del riesgo, riesgo volcánico, vulnerabilidad, mapa mental, incertidumbre, manejo de crisis, preparación, prevención, Cotopaxi, Valle de Los Chillos

* Equipo de investigación PAUD (Por el Ambiente Urbano y Desarrollo), García Moreno 751 y Sucre -Ex Hogar Javier-. Edificio Dirección Metropolitana de Planificación Territorial, Quito, Ecuador. Becaria el IFEA (UMIFRE 17, CNRS-MAEE) en 2008. E-mail: dia_salazar@hotmail.com

** Institut de Recherche pour le Développement (IRD), UR 029, programa Pacivur, Calle Teruel 357, Miraflores, Casilla 18-1209, Lima 18, Perú. E-mail: robert.dercole@ird.fr 


\title{
Perception du risque lié au volcan Cotopaxi et vulnérabilité dans la vallée de Los Chillos (Équateur)
}

\section{Résumé}

Une enquête sur la perception du risque lié au volcan Cotopaxi a été réalisée en 2007 dans la vallée de Los Chillos, située à proximité de Quito. L'analyse porte sur la perception de l'aléa volcanique et ses caractéristiques, la perception des espaces les plus exposés aux phénomènes volcaniques et la perception du risque au lieu de résidence. Les résultats de l'enquête mettent en évidence une conscience claire de l'existence du risque volcanique, mais également des distorsions significatives entre le point de vue des scientifiques et celui de la population sur les zones les plus exposées. Compte tenu des incertitudes qui caractérisent le risque volcanique, ce type de recherche sur la perception des risques est très utile en termes d'action préventive et de réduction de la vulnérabilité de la population. Son objectif n'est pas de rallier le maximum de population à un point de vue unique, jugé objectif, celui des scientifiques, mais de montrer l'intérêt de relier de manière constructive les points de vue des différents acteurs.

Mots clés : perception du risque, risque volcanique, vulnérabilité, carte mentale, incertitude, gestion de crise, préparation, prévention, Cotopaxi, Valle de Los Chillos

\section{Cotopaxi volcano risk perception and vulnerability in Los Chillos Valley (Ecuador)}

\begin{abstract}
A survey on Cotopaxi volcano risk perception was conducted in 2007 in Los Chillos Valley located near Quito. The analysis focuses (1) on perception of volcanic hazards and its characteristics, (2) on perception of the most exposed areas to volcanic hazards and (3) risk perception in the place of residence. The survey results show a clear awareness of the existence of volcanic hazard. It also shows significant distortions between the scientists and population points of view on the most exposed areas. Given the uncertainties in volcanic risk, this type of research on risk perception is very useful in terms of prevention and population vulnerability reduction. Its objective is not to ensure that people have the same view that the scientists but to demonstrate the benefit of linking constructively the views of different actors.
\end{abstract}

Key words: risk perception, volcanic hazard, vulnerability, mental map, uncertainty, crisis management, preparing, prevention, Cotopaxi, Valle de Los Chillos

\section{INTRODUCCIÓN}

Para las ciencias sociales, el riesgo no puede reducirse a una dimensión técnica como la del cálculo de probabilidades. Este depende de quien lo percibe y es, en consecuencia, una construcción social, una percepción variable según los grupos y los individuos (Veyret et al., 2004). La percepción del riesgo'1 es un proceso

1 En este artículo hablaremos indistintamente de percepción del riesgo y de representación del riesgo, aunque en realidad existe una distinción entre ambos conceptos. En efecto, la percepción es la 
por el cual el individuo organiza e interpreta sus impresiones sensoriales a fin de dar un sentido a su medio ambiente (Zayed, 2006). Está moldeada por factores psicológicos que explican particularmente por qué algunos riesgos producen más ansiedad que otros (Slovic, 1987). Está influida por la visión del mundo del sujeto, la misma que también está marcada por la cultura en general y la estructura social en particular (Douglas, 1970; Douglas \& Wildavsky, 1982). Estos últimos autores insisten en que nuestras percepciones varían en función de nuestra pertenencia a determinados grupos sociales y que solemos establecer una jerarquía entre las diferentes amenazas según valores que caracterizan formas sociales diferentes (Veyret et al., 2004). Como toda forma de organización social tiene sus propios valores, también tendrá su propia manera de jerarquizar los riesgos, su propia «cartera de riesgos» (Peretti-Watel, 2003).

De manera general, entonces, identificar los factores que tienen una incidencia sobre la manera de percibir el riesgo es una tarea compleja, dada su diversidad: nivel educativo, categoría socioeconómica, actividad profesional, aspectos familiares, culturales y étnicos, experiencia anterior en materia de desastre, etc. (Alexander, 2000). Los medios de comunicación desempeñan también un rol importante, pues no solo modifican la representación de los riesgos, sino que también contribuyen a su construcción social y a su representación mental (Dauphiné, 2001).

La percepción del riesgo es igualmente variable según el peso de la competencia entre riesgos (Alexander, 2000): por ejemplo, el peligro de perder el empleo y por ende la fuente de ingresos, puede reducir de manera importante el grado de percepción del riesgo sísmico o de inundaciones.

En este contexto, los estudios de percepción de riesgo permiten entender cómo la población, ya sea de modo individual o colectivo, observa el mundo, su entorno, el espacio donde vive y sus problemas (D'Ercole, 2008). Sus autores examinan el discernimiento de la gente cuando se les pide caracterizar y evaluar peligros o actividades peligrosas (Slovic, 1987). Estos estudios son realizados por las ciencias humanas y sociales como la sociología, ciencias políticas, antropología, psicología, y también por la geografía, disciplina de los autores de este artículo.

El enfoque geográfico se distingue del de otras ciencias sociales. Como los sociólogos, los geógrafos se consagran a las representaciones colectivas, más allá de aquellas que tienen que ver con las entidades sociales, para considerar el espacio y la manera como este se valoriza (Claval, 1992). Se estudia a los diferentes actores y sus modos de representación del riesgo no como tales, sino en tanto su incidencia sobre la territorialización del riesgo y la organización espacial (Veyret et al., 2004).

El objetivo de estas investigaciones es alimentar los estudios de riesgo y ayudar en la elaboración de políticas de reducción de los riesgos y de las vulnerabilidades, proveyendo las bases para comprender y anticipar las respuestas del público a

función mediante la cual la mente se representa objetos en su presencia, mientras la representación permite evocar objetos aun cuando estos no son directamente perceptibles (Bailly, 1992), siendo este el caso del riesgo. 
las situaciones de crisis (Slovic, 1987). Facilitan también la comunicación entre la población, los científicos y los responsables políticos. En el campo de los riesgos, sean estos de origen volcánico o de otro tipo, la población no comparte uniformemente la visión de los científicos. Los vulcanólogos, sismólogos o hidrólogos tienen su propia percepción, basada en criterios científicos, mientras que la población funda su percepción en criterios propios relacionados con la cultura, la religión, las características socioeconómicas y de educación, el tiempo de residencia en un lugar, las experiencias anteriores, la edad, el género, etc. Al mismo tiempo, las representaciones que los individuos se hacen de su situación influyen sobre sus reacciones y sus comportamientos (Claval, 1992). El modo en que la población percibe las amenazas y los riesgos puede inducir comportamientos peligrosos, tanto para los individuos como para la comunidad, o por lo menos muy diferentes a los esperados por los científicos y autoridades políticas, lo cual obviamente dificulta el manejo de crisis. Sin embargo, no es tanto la percepción de la población la que plantea problemas, sino más bien la distancia entre esta y la de los científicos; es sobre todo este desfase el que debe ser utilizado para la elaboración de los planes de emergencia.

En este artículo tratamos de la percepción del riesgo volcánico, tema que ya ha congregado a cierto número de investigadores de diferentes disciplinas (Perry et al., 1983; D'Ercole, 1991; D'Ercole \& Rançon, 1994; D'Ercole \& Rançon, 1999; D'Ercole \& Metzger, 2002; Davis \& Ricci, 2004; Dominey-Howes \& MinosMinopoulos, 2004; Lavigne et al., 2008; etc.). Tres aspectos de la percepción de los riesgos constituyen la estructura del artículo. Por un lado, la percepción de la amenaza volcánica y de sus características (tipos de fenómenos, probabilidad de ocurrencia, evolución en el tiempo de una erupción, magnitud de la erupción). Por otro lado, la percepción de los espacios más expuestos a los fenómenos volcánicos. Y por último, la percepción misma del riesgo; en otros términos, la percepción del nivel de consecuencias que traería una erupción a las personas y su lugar de residencia. El artículo comienza con la presentación de la encuesta efectuada en el Valle de Los Chillos (Quito) y de la metodología empleada. Concluye con una reflexión sobre el aporte de este tipo de investigación en términos de reducción de la vulnerabilidad de la población amenazada.

\section{CONTEXTO DE LA INVESTIGACIÓN Y METODOLOGÍA}

La investigación sobre la percepción del riesgo volcánico² ha sido desarrollada en el Valle de Los Chillos, una gran hondonada ubicada al suroriente de la ciudad de Quito (fig. 1), al límite del cantón Quito (correspondiente al territorio del Distrito Metropolitano de Quito) y del cantón Rumiñahui, cuya capital es Sangolquí. El Valle

2 La investigación fue realizada por Diana Salazar, bajo la dirección de Robert D’Ercole, en el marco del programa Pacivur (Programa andino de capacitación e investigación sobre vulnerabilidad y riesgos en medio urbano) y de la preparación de una tesis de ingeniería geográfica, cuya defensa tuvo lugar en marzo del 2008 en la PUCE (Pontificia Universidad Católica del Ecuador). 


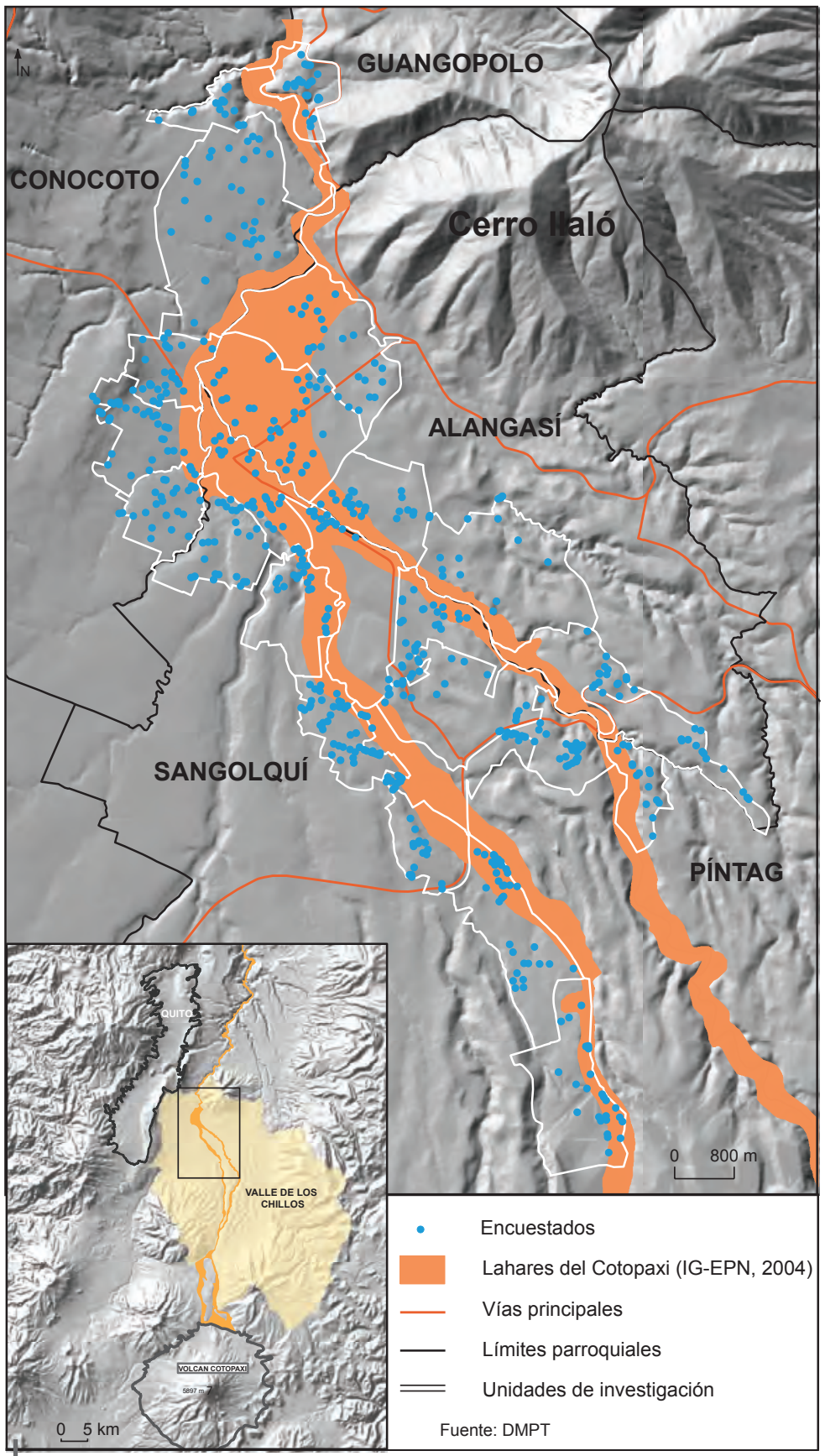

Figura 1 - Las bases territoriales de la encuesta

Fuente: Salazar, 2008 
comprende una aglomeración urbana continua de más de 180000 habitantes, localizada preferentemente en las riberas de los ríos Pita y Santa Clara. Estos ríos, tranquilos y agradables en tiempo normal, constituyen la vía de paso del peligro más grave: el peligro asociado a los lahares ${ }^{3}$ que se producirían con una erupción del volcán Cotopaxi (5 897 msnm), localizado a unos 60 km al sur del límite norte del Valle de Los Chillos (fig. 2). Los lahares, ligados al derretimiento por lo menos parcial de su casquete glaciar, pondrían directamente en peligro a más de 15000 habitantes si el fenómeno ocurriera de noche y cerca de 50000 personas en caso de ocurrir de día (Robert, 2007). El Cotopaxi ha tenido 9 erupciones de gran magnitud 4 desde 1534 . La última erupción que produjo un lahar importante fue en junio de 1877, el cual destruyó gran parte del Valles.

Entre 1987 y 1990, R. D’Ercole realizó una investigación sobre la vulnerabilidad de la población expuesta al volcán Cotopaxi sobre la base de una encuesta efectuada a 2200 personas (D’Ercole, 1991). Él demostró que en el Valle de los Chillos existían los niveles más bajos de percepción de riesgo con relación a toda el área de investigación (que comprendía la Provincia de Pichincha, al norte y la Provincia de Cotopaxi, al sur). Casi veinte años después, en 2007, basándose igualmente en una encuesta, se inició un estudio de percepción de riesgos de la población del Valle de los Chillos en el marco de una colaboración entre el programa Pacivur del IRD y la Casa Cotopaxí. El área de estudio comprendió parte de las cinco parroquias (Alangasí, Conocoto, Guanguapolo, Sangolquí y Píntag) del Valle que, según los vulcanólogos, se verían afectadas por el paso de los lahares (fig. 1). De un total de 8432 viviendas, se construyó una muestra de 615 a ser encuestadas. Para facilitar la muestra, la cartografía y el análisis de informaciones provenientes de la encuesta, se dividió el área de estudio en 25 unidades de investigación, delimitadas tomando en cuenta los siguientes criterios: límites de la zona de mayor peligro de lahares establecidos por el Instituto Geofísico de la Escuela Politécnica Nacional, y un área de 500 a 1000 m más allá de esta zona7; límites naturales (ríos) y administrativos (parroquias y cantones); sectores censales del Instituto Nacional de Estadísticas y Censos (INEC); uso de suelo y distribución de la población.

La encuesta aplicó un cuestionario de 28 preguntas a 615 habitantes de la zona, además de un mapa base donde cada encuestado señaló las zonas consideradas,

3 Flujos de lodo y escombros, mezclas de materiales volcánicos saturados de agua y movilizados.

4 Con VEl (Índice de Explosividad Volcánica) igual o superior a 4 según una escala que va de 0 (erupción no explosiva) a 8 (erupción extremadamente explosiva).

5 Para mayor información sobre el Valle de Los Chillos y sobre los peligros asociados al volcán Cotopaxi, ver el artículo de J. Robert et al. en este volumen (pp. 709-733).

6 Centro de información en gestión de riesgos de la Unidad de Gestión de Riesgos de la Administración Zonal Valle de los Chillos (AZVCH). La AZVCH, creada en 1997, es una de las ocho administraciones zonales del Distrito Metropolitano de Quito. Está conformada por seis parroquias: Guangopolo, Conocoto, Alangasí, La Merced, Amaguaña y Píntag.

7 La idea fue no restringirse a los espacios considerados por los vulcanólogos como de mayor riesgo, debido en particular a los problemas planteados por la delimitación de tales espacios (ver el artículo de J. Robert et al. en este volumen, pp. 709-733). 


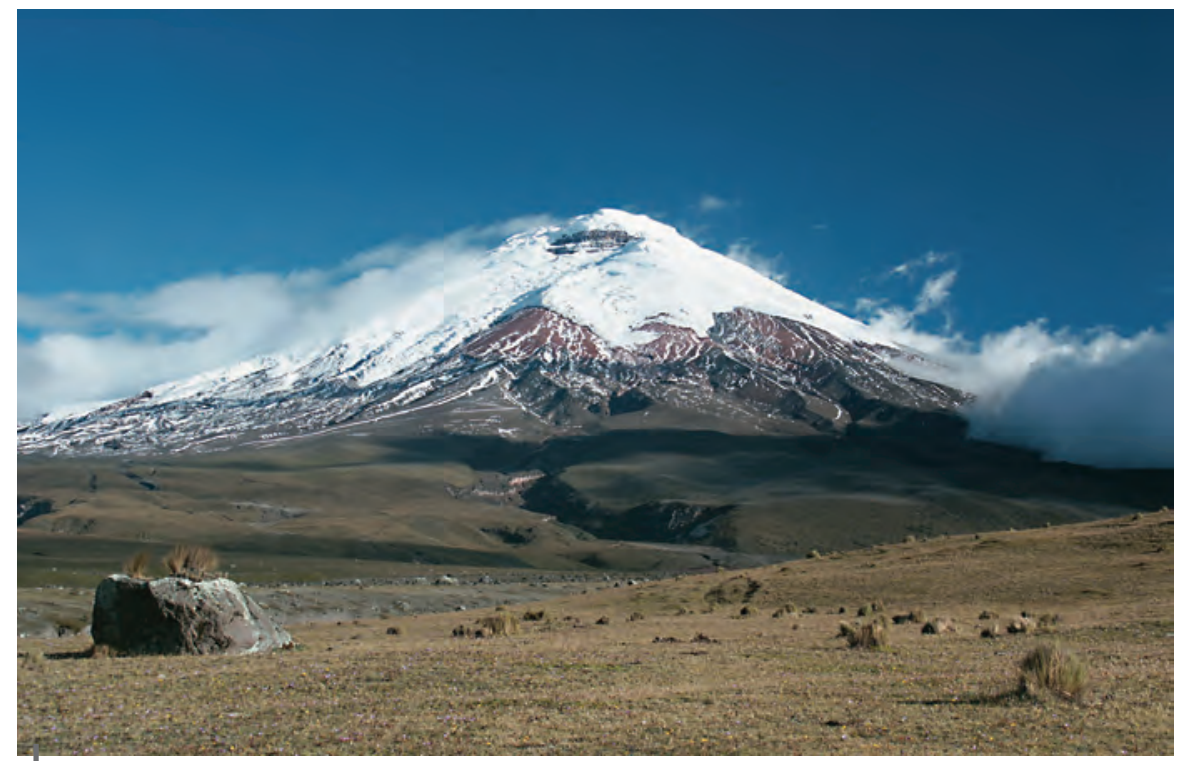

Figura 2 - El volcán Cotopaxi (5 897 msnm) visto desde el noroeste Foto: J. Robert, 2007

por él mismo, peligrosas ante una erupción del Cotopaxi. Con los datos así levantados se construyó una base de datos tratada a través del programa Statistical Package for Social Sciences (SPSS). En un Sistema de Información Geográfica (SAVGIS8) se georeferenció un punto para cada hogar encuestado, calificado con las respuestas a las preguntas (fig. 1). Asimismo, se relacionó la base de datos con los puntos, representando los hogares y de ahí las unidades de investigación. Esto permitió elaborar mapas temáticos según las preguntas realizadas, facilitando el análisis espacial de los resultados.

\section{PERCEPCIÓN DE LOS PELIGROS VOLCÁNICOS EN EL VALLE DE LOS CHILLOS}

Se considera aquí la percepción de los fenómenos físicos (peligros o amenazas), producto de la erupción volcánica y de sus características.

\section{1. Percepción de los peligros naturales que amenazan el Valle}

De los 615 encuestados, el 84 \% nombró a las erupciones volcánicas como el principal peligro natural que amenaza el Valle. Las otras amenazas vienen mucho

8 SAVGIS es un sistema de información geográfica desarrollado por el IRD (concepción Marc Souris). 
después (28 \% de las personas interrogadas citan las inundaciones, 12 \% los sismos y $5 \%$ los deslizamientos de tierra y los aluviones [o huaycos]). Este alto porcentaje se debe a que, desde 1999 en Ecuador, varios volcanes han reiniciado su actividad después de muchos años de haber permanecido en calma. Primeramente, a proximidad de la ciudad de Baños, el volcán Tungurahua entró en actividad a mediados de septiembre de 1999 y permanece así hasta la actualidad, provocando daños materiales y económicos cuantiosos, así como ciertas pérdidas humanas (3 muertos en 2006). En el mismo año, después de un año de actividad freática, se reactivó el Guagua Pichincha, vecino de la ciudad de Quito, que hizo sentir los estragos de la ceniza a los quiteños. La puesta en alerta volcánica de la ciudad y la erupción de este volcán provocaron por primera vez una reflexión acerca de este peligro por parte de la población y de las instituciones. En 2002, el volcán Reventador, ubicado a un centenar de kilómetros de Quito, en la zona subandina, hizo revivir a la población quiteña los efectos de la ceniza.

De los 517 interrogados que citaron a las erupciones volcánicas como principal amenaza para el Valle, el 81 \% nombró al volcán Cotopaxi, a pesar de que la última erupción de este volcán ocurrió en el siglo XIX. Este alto porcentaje puede deberse a que, en 2002, una actividad anómala, evidenciada a través de sismos y fumarolas, provocó que las autoridades alertaran a la población sobre su posible reactivación. Es así que, tanto la experiencia vivida con los volcanes Tungurahua, Pichincha y Reventador en los últimos años como la alerta del Cotopaxi entrando en actividad, han generado mayor conciencia en la población del Valle de los Chillos y del país en general, acerca del peligro volcánico al que está expuesta.

\section{2. El Cotopaxi como fuente de peligro}

El análisis de las respuestas a la pregunta sobre lo que representa el Cotopaxi, muestra que este volcán está lejos de dejar indiferente a la población. Cerca del $70 \%$ de los interrogados lo ve como un peligro o una fuente de inquietud y el $40 \%$ lo ve solamente, o al mismo tiempo, de manera muy positiva. A pesar de la inquietud de la mayoría, esto refleja que existen muchas personas que han aprendido a vivir con el volcán sin temerle y que, al contrario, lo consideran beneficioso por ser uno de los principales atractivos turísticos del país y la principal fuente de agua para el Valle de los Chillos. Finalmente, pocas personas (apenas más del 10 \%) muestran un punto de vista neutro sobre el volcán. 
Cuadro 1 - Lo que representa el Cotopaxi para los encuestados

\begin{tabular}{|c|c|c|c|}
\hline \multicolumn{2}{|r|}{$\begin{array}{l}\text { Respuesta a la pregunta: } \\
\text { ¿Qué representa para Ud. el Cotopaxi? }\end{array}$} & \multirow{2}{*}{\begin{tabular}{|r|}
$\begin{array}{c}\text { Frecuencia } \\
\text { de } \\
\text { respuestas* }\end{array}$ \\
399 \\
\end{tabular}} & \multirow{2}{*}{$\begin{array}{r}\begin{array}{c}\text { \% en } \\
\text { relación al } \\
\text { número de } \\
\text { encuestas }\end{array} \\
64,9\end{array}$} \\
\hline & Fuente de peligro & & \\
\hline $\begin{array}{l}\text { Vision } \\
\text { negativa }\end{array}$ & $\begin{array}{l}\text { Sentimientos negativos o de alerta } \\
\text { (preocupación, temor, angustia, inseguridad, } \\
\text { incertidumbre) }\end{array}$ & 60 & 9,7 \\
\hline \multirow{5}{*}{$\begin{array}{l}\text { Visión } \\
\text { positiva }\end{array}$} & Belleza, majestuosidad de paisaje & 161 & 26,2 \\
\hline & Atractivo turístico/recreación & 54 & 8,8 \\
\hline & Fuente de beneficios, de vida, reserva natural & 25 & 4,1 \\
\hline & $\begin{array}{l}\text { Sentimientos positivos (admiración, respeto, } \\
\text { placer, protección) }\end{array}$ & 18 & 2,9 \\
\hline & Símbolo nacional & 7 & 1,1 \\
\hline \multirow{2}{*}{$\begin{array}{l}\text { Visión } \\
\text { neutra }\end{array}$} & Definición únicamente neutra** & 62 & 10,1 \\
\hline & Nada/No sabe & 7 & 1,1 \\
\hline \multicolumn{2}{|r|}{ Visión negativa sola } & 306 & 49,8 \\
\hline \multicolumn{2}{|r|}{ Visión positiva sola } & 123 & 20,0 \\
\hline \multicolumn{2}{|r|}{ Visión negativa y positiva } & 117 & 19,0 \\
\hline \multicolumn{2}{|r|}{ Visión únicamente neutra } & 69 & 11,2 \\
\hline \multicolumn{2}{|r|}{ Total } & 615 & 100 \\
\hline
\end{tabular}

Fuente: D. Salazar, 2008

* Múltiples respuestas para un solo encuestado fueron posibles.

** Se consideran como «neutras» aquellas respuestas que no tienen ninguna connotación de peligro, perjuicio o de beneficios. El Cotopaxi es considerado únicamente como un volcán, un nevado o una montaña.

\section{3. Percepción de los fenómenos volcánicos que amenazan el Valle}

Un poco más del 50 \% de los encuestados nombró a la ceniza volcánica como principal fenómeno volcánico relacionado al Cotopaxi (fig. 3). Esto se puede explicar por las erupciones recientes del Pichincha y del Reventador, donde la ceniza ha sido el principal material que llegó a la capital y sus alrededores. Una proporción equivalente de entrevistados piensa que, en caso de una erupción, 


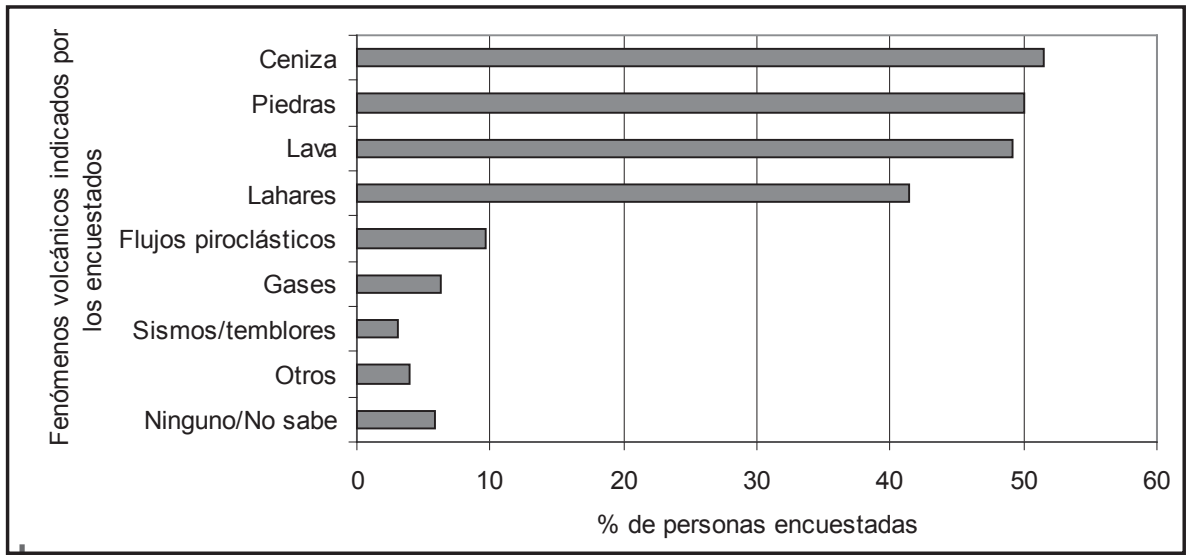

Figura 3 - Fenómenos volcánicos del Cotopaxi que amenazan el Valle de Los Chillos, según los encuestados

Fuente: D. Salazar, 2008

tanto las piedras como la lava podrían llegar hasta el Valle. Las piedras se encuentran efectivamente dispersas en casi todo el Valle y sus habitantes saben que son producto de erupciones del Cotopaxi (sin por ello estar de acuerdo sobre la manera cómo estas piedras fueron transportadas y depositadas9), razón por la cual también son nombradas con frecuencia. En lo que atañe a la lava, la percepción parece totalmente contraria a la del Instituto Geofísico (IG-EPN) según el cual su extensión sería únicamente a nivel de la zona cercana al cráter, hasta los flancos o laderas del volcán.

De manera sorprendente, únicamente el $41 \%$ considera a los lahares10 como una amenaza. El porcentaje es relativamente bajo si consideramos que éste es uno de los principales peligros asociados al volcán Cotopaxi, según los científicos. Los flujos piroclásticos, los gases y sismos o temblores son los fenómenos menos nombrados por la población, ya que no alcanzan ni el $10 \%$. Otros fenómenos o materiales expulsados como fuego, candela, escombros, arena y cobre fueron nombrados por el $4 \%$ de la población. Únicamente el $6 \%$ de los interrogados no pudo pronunciarse al respecto, ya sea por desconocimiento o porque consideró que no existía ninguna amenaza.

9 Algunas personas dicen que las piedras fueron transportadas por los ríos y otras consideran que fueron lanzadas directamente desde el volcán.

10 No todas las personas encuestadas se referían estrictamente al término «lahares», razón por la cual otras denominaciones (sinónimo de este fenómeno) también fueron consideradas: deslaves, inundaciones, aluviones, aludes. 


\section{4. Percepción de la probabilidad de ocurrencia de una erupción del volcán Cotopaxi y de su desarrollo en el tiempo}

En cuanto a la posibilidad de ocurrencia de una erupción del Cotopaxi, de los 615 encuestados, el 85 \% considera que el volcán sí va a erupcionar, mientras que el $10 \%$ opina lo contrario; el $5 \%$ restante corresponde a aquellas personas indecisas o que no respondieron la pregunta. Se tiene entonces una percepción clara de un volcán que es capaz de entrar en erupción.

Por otro lado, cuando se les pregunta a los encuestados sobre cuándo va a erupcionar el volcán, el 58,4 \% responde que en cualquier momento; es decir, podría ser tanto el día de mañana como dentro de muchos años (fig. 4). Un 23,6 \% tiene una idea más precisa del momento de la erupción; de ellos, el 1,3 \% afirma que la erupción del volcán es a corto plazo, es decir en menos de un año; el $11,5 \%$ piensa que este podría erupcionar entre uno y diez años (mediano plazo); y el 10,7 \% considera que la erupción del volcán tendrá lugar en el largo plazo (dentro de 10 a 50 años).

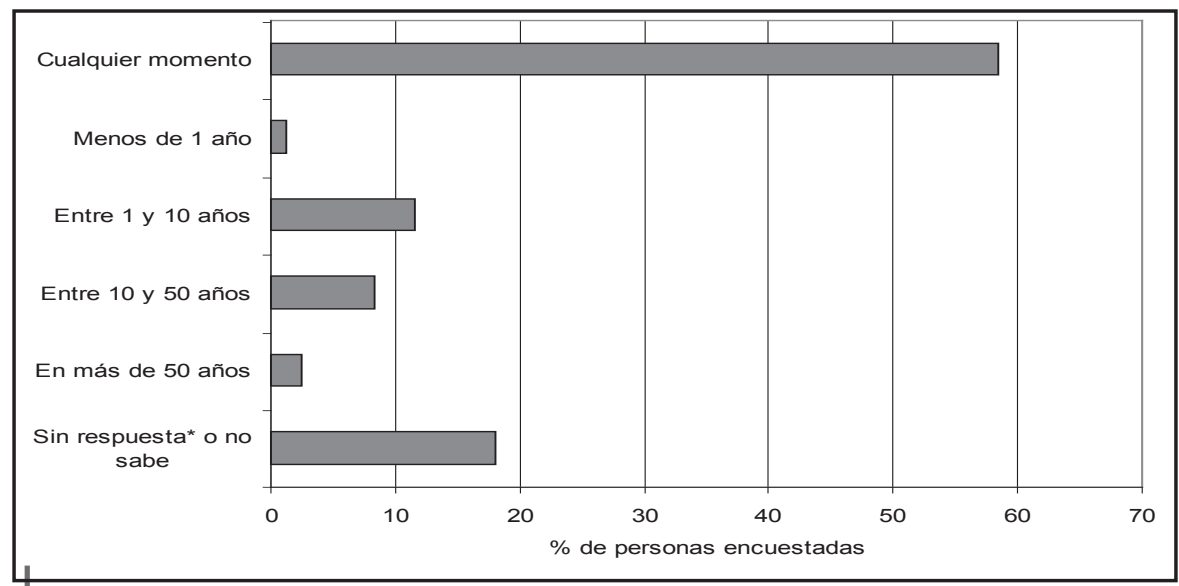

Figura 4 - Cuándo puede ocurrir la próxima erupción del Cotopaxi, según los encuestados

Fuente: D. Salazar, 2008

\footnotetext{
*Los encuestados sin respuesta (aproximadamente $15 \%$ ) corresponden a aquellas personas que anteriormente habían respondido que el volcán no iba a erupcionar o que no saben responder si esto va a ocurrir.
}

Comparando los resultados con aquellos de R. D’Ercole (D'Ercole, 1991), se observa que en aquel entonces un alto porcentaje de personas (86 \%) creía que la erupción del Cotopaxi ocurriría en los próximos diez años. Actualmente, la población expuesta parece tener las ideas menos claras en cuanto a la fecha de la próxima erupción: solamente un 11 \% piensa que la erupción puede producirse en los 10 años venideros. En cambio, la importancia del número de respuestas «en 
cualquier momento» muestra que la población ha madurado. Si bien considera que una erupción es bastante posible, sabe también que es difícil prever el momento preciso en que se producirá, rompiendo así con las certezas que se tenían hace 20 años. Las erupciones volcánicas recientes y la incertidumbre que las ha acompañado11 han servido de lección, así como también la alerta sobre la reactivación del Cotopaxi en el 2002. Varias personas encuestadas cuentan cómo numerosas familias creyeron en una erupción inminente del Cotopaxi, lo que tuvo como consecuencia una fuerte inquietud, la venta de sus lotes a precios irrisorios y el abandono de ciertas viviendas que se pudo evidenciar en el campo. Sin embargo, de esto han pasado ya varios años y el volcán no ha erupcionado, por lo cual la población ya no se aventura a prever la próxima erupción.

Con respecto al desarrollo en el tiempo de una erupción (fig. 5), el 16,7 \% de los interrogados piensa que pasarían algunos meses —o incluso años — antes de que se presente una erupción, una vez que el volcán se active. El 6,2 \% afirma que, a pesar de que el volcán manifieste una actividad, no necesariamente significa que habrá una erupción (o por lo menos una erupción importante); para el 5,2% de los encuestados, no es posible predecir la temporalidad de la erupción. El 35,6 \% de los encuestados, en cambio, considera que una vez activado el volcán, se producirá una erupción repentina (segundos, minutos, horas, días, semanas).

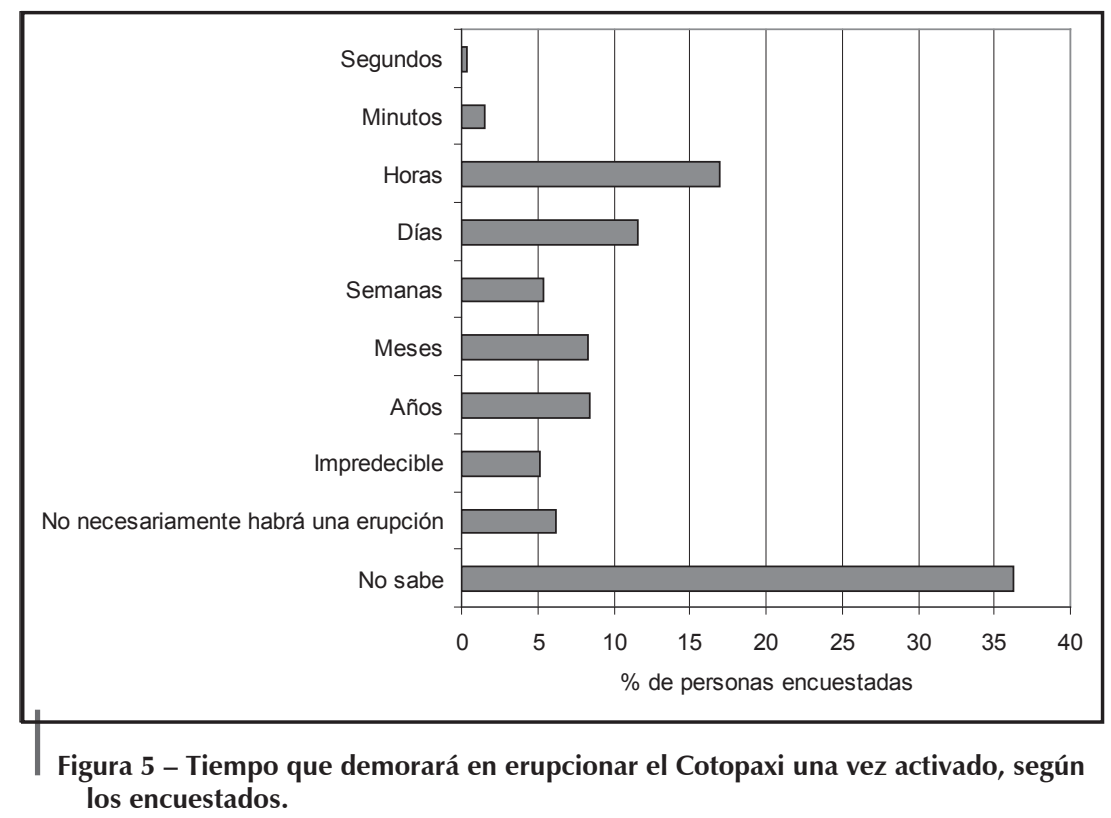

Fuente: D. Salazar, 2008

11 Largo periodo eruptivo del Tungurahua marcado por erupciones de magnitudes desiguales; un año de actividad freática del volcán Pichincha antes de pasar al estadio magmático; corto periodo eruptivo del volcán Reventador. 
Esta manera de pensar puede reflejarse en comportamientos semejantes a los de 2002, o incluso peores, en caso de pánico colectivo. Así mismo, existe un 36,3 \% de encuestados que no tiene idea del tema, lo que también podría suponer problemas, en la medida en que su conducta es aún más incierta.

\section{5. Percepción de la magnitud de una erupción del Cotopaxi y de su alcance geográfico}

Con respecto a la magnitud de una erupción del Cotopaxi, el 68 \% de la población encuestada considera que sería de gran tamaño (fig. 6). Apenas el $5 \%$ piensa que sería pequeña, mientras que el $11 \%$ piensa que sería de tamaño mediano. El $16 \%$ no tiene idea de la magnitud posible de una erupción del volcán. Se observa, en general, una fuerte y previsible preocupación de la población, una vez se reactive el volcán.

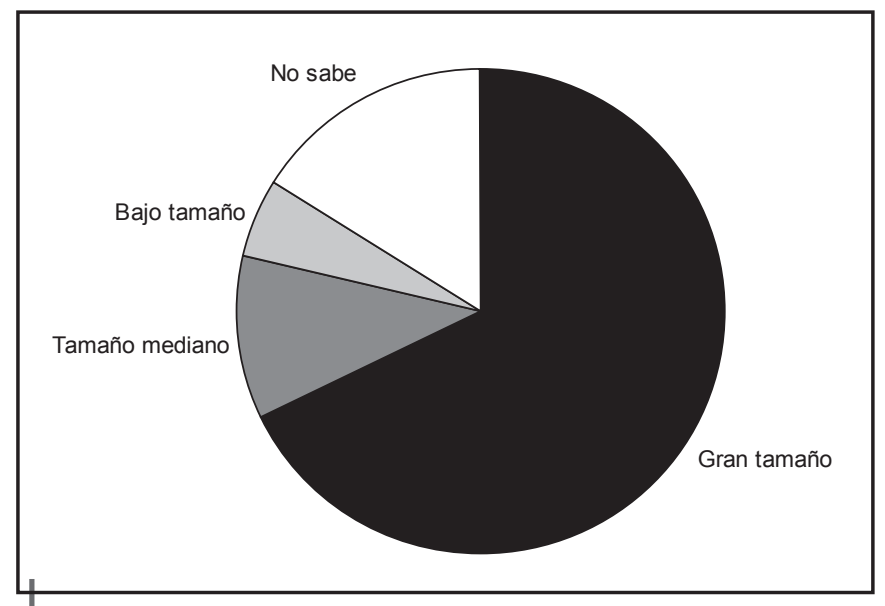

Figura 6 - El tamaño de una erupción del Cotopaxi según los encuestados

Fuente: D. Salazar, 2008

Por otra parte, el 78\% de los encuestados opina que el alcance geográfico de una eventual erupción sería de nivel multiprovincial (se nombró sobre todo a las provincias de Cotopaxi y Pichincha, a las que se agrega frecuentemente la de Tungurahua) hasta nacional. El 7 \% piensa que el impacto de una erupción se daría únicamente en las faldas del volcán, mientras que el $9 \%$ afirma que el alcance solamente sería a nivel de una provincia. Dada la amplitud que se le otorga al territorio que podría ser afectado, nuevamente se hace evidente entre los encuestados el temor a una erupción de gran magnitud. 


\section{PERCEPCIÓN DE LOS ESPACIOS MÁS EXPUESTOS AL PELIGRO VOLCÁNICO}

La percepción de los espacios expuestos al peligro es una etapa intermedia en el análisis entre la percepción de los fenómenos destructores (amenazas) y la percepción de los riesgos. En efecto, esta última está directa o indirectamente ligada a los lugares que se perciben como posibles afectados por fenómenos destructores. Su análisis permite identificar los puntos de vista de la población amenazada sobre el tema, considerados generalmente como subjetivos, y compararlos con aquellos de los especialistas (los vulcanólogos en lo que nos atañe) que se suponen más objetivos. La comparación permite evidenciar problemas susceptibles de plantearse en periodo de emergencia, en razón de la diferencia de los puntos de vista y de un manejo de crisis que se funda en la visión de los científicos.

Para hacerlo, hemos optado por el mapa mental de los espacios más expuestos a los fenómenos volcánicos. Como el organismo humano es profundamente adaptable y flexible, las personas que pertenecen a grupos diferentes pueden hacerse una idea muy diferente de la misma realidad exterior (Lynch, 1960). Esta imagen mental, que es una forma particular de representación de la realidad, puede traducirse mediante la representación gráfica de un espacio por una persona. El mapa mental, usado generalmente por los geógrafos, es utilizado para dar una idea de la manera cómo diferentes individuos o grupos sociales perciben su medio ambiente (Peake, 2004).

El urbanista norteamericano Kevin Lynch, con su obra The image of the city (Lynch, 1960), puede ser considerado como el precursor de los mapas mentales. En ese libro, el autor muestra que la imagen que los habitantes de Boston tienen de su ciudad, de sus elementos estructurantes, puede presentar diferencias muy importantes frente a la visión de los especialistas (como los urbanistas). Lynch muestra que el conocimiento de esta percepción, que se puede visualizar con mapas mentales realizados a partir de entrevistas, permite al urbanista, al arquitecto o al sociólogo mejorar de modo substancial el ordenamiento, la composición urbana y la calidad de vida de los habitantes. Desde aquellos estudios precursores, varios geógrafos han utilizado y desarrollado el concepto de mapa mental, como Peter Gould (Gould, 1966; Gould \& White, 1974) o Antoine Bailly (Bailly, 1987; Bailly et al., 1995). El concepto y la metodología fueron adaptados a inicios de los años 1990 por Robert D'Ercole en el campo de la percepción de los riesgos. Así, se han realizado mapas mentales de los espacios expuestos al volcán Montaña Pelée en la isla de la Martinica (D'Ercole \& Rançon, 1994; D’Ercole \& Rançon, 1999), y de los espacios amenazados por el volcán Pichincha en Ecuador (D'Ercole \& Metzger, 2002).

La metodología desarrollada por R. D’Ercole se implementó en el Valle de los Chillos y consistió en presentar al encuestado un mapa con referencias espaciales básicas: ríos, vías principales, trazado urbano y los nombres de los lugares principales. Una vez ubicada la vivienda del interrogado en el mapa, se le solicitó graficar aquellos lugares que, según él, se verían más afectados por productos volcánicos en caso de 
una erupción del Cotopaxi12. Esto permitió llegar a una cartografía de los espacios considerados como los más expuestos para las personas entrevistadas. De esta manera se obtuvieron gráficos que fueron ingresados a un SIG y sobrepuestos para así obtener un mapa sintético. Las clases representan el porcentaje de personas que señalaron tal o cual punto del espacio (fig. 7): la clase 91-100\%

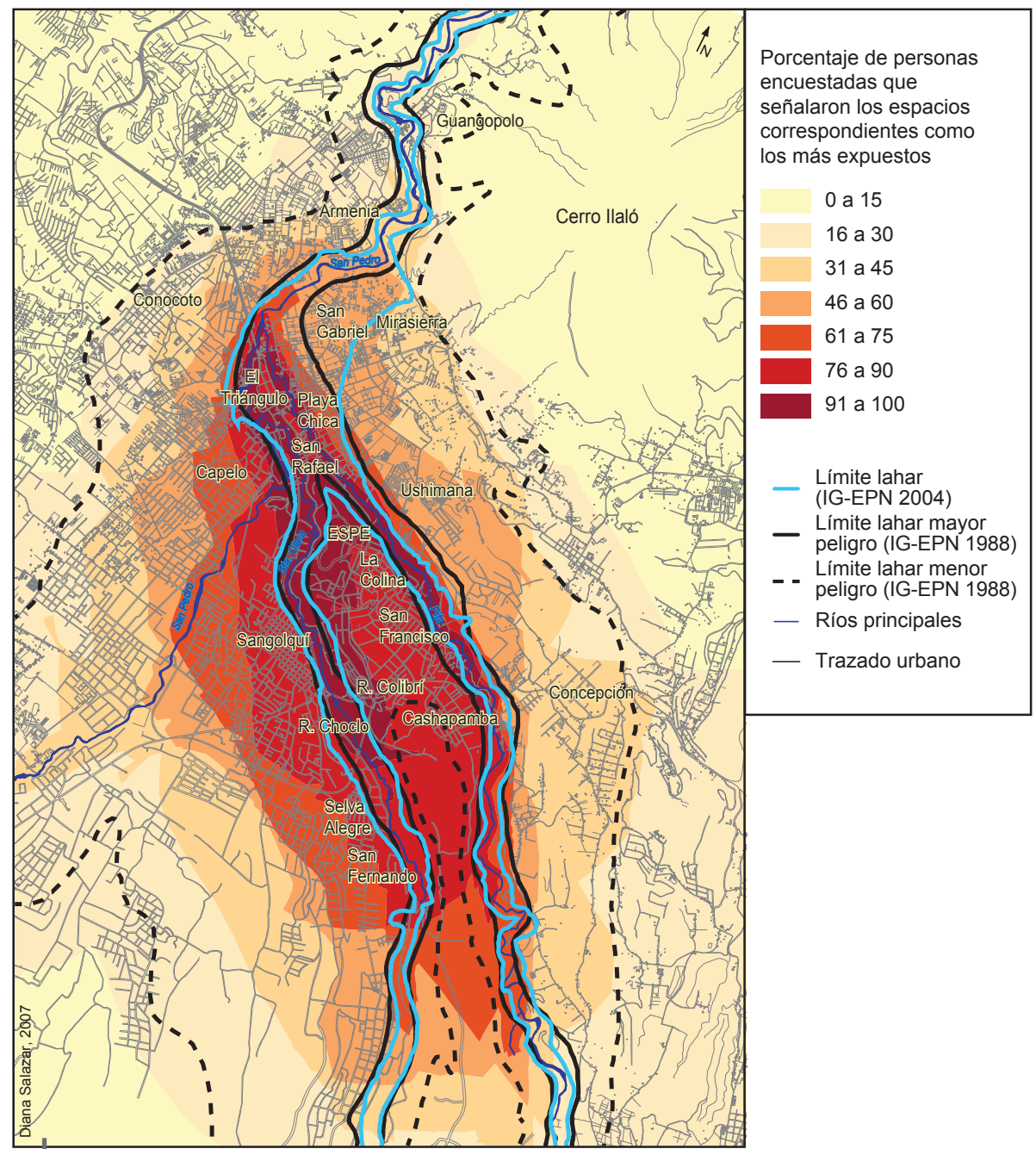

Figura 7 - Lugares más expuestos a una erupción del volcán Cotopaxi, según los encuestados

Realización: D. Salazar

12 Algunas personas interrogadas solo tenían una cultura limitada del mapa o de las escalas geográficas. En este caso, el encuestador ayudó a la persona interrogada a ubicarse en el documento cartográfico y a identificar las zonas deseadas. 
representa los espacios considerados como muy peligrosos por una gran mayoría de la población; a la inversa, la clase 0-15 \% señala los espacios considerados no peligrosos o poco peligrosos por la mayoría de los interrogados. A este mapa se añadieron los límites de los lahares establecidos por el IG-EPN en 1988 y 2004, a fin de observar diferencias entre los espacios de la amenaza considerados por los científicos y las representaciones del peligro por los encuestados.

La figura 7, que presenta el punto de vista del conjunto de la muestra13, ofrece una visión parcialmente distinta entre la población y los científicos en lo que atañe a las zonas más expuestas al volcán Cotopaxi.

De sur a norte, las zonas consideradas como más expuestas por la población se sitúan de manera aproximada entre el lugar denominado «San Francisco»y la zona del «Triángulo», donde se encuentran los ríos Pita y Santa Clara. Según los científicos, en cambio, los sectores expuestos a los lahares comienzan a nivel del cráter del volcán situado al sur, a más de 40 km del lugar denominado «San Francisco», y se prolongan hacia el norte. La interrupción en la representación de las zonas expuestas hacia el norte puede comprenderse en la medida en que la población estima que la exposición disminuye, habida cuenta la distancia del volcán y el angostamiento del valle. Hacia el sur, en dirección del volcán, la interrupción es más sorprendente y denota verosímilmente cierto desconocimiento de los espacios muy poco poblados y poco accesibles que se sitúan hasta el pie del volcán. Esto refleja también que las personas interrogadas han querido representar espacios expuestos a fenómenos volcánicos que sean, al mismo tiempo, espacios de riesgo; es decir, lugares que tienen población, bienes y actividades. Los espacios no poblados han sido, por ende, excluidos.

De este a oeste, el peligro disminuye a medida que uno se aleja de los ríos y de su zona de confluencia, lo que es lógico en la perspectiva de ocurrencia de lahares. Sin embargo, los interrogados señalan zonas fuertemente expuestas mucho más extensas que las de los vulcanólogos, por lo menos según el mapa elaborado en 2004 por el Instituto Geofísico. Por ejemplo, pocos consideran el sector ubicado entre los ríos Pita y Santa Clara como seguro, mientras que los vulcanólogos estiman que los lahares no lo afectarían. Ocurre lo mismo hacia el oeste, en dirección de Conocoto y Sangolquí, donde las zonas consideradas como muy peligrosas se alejan más de un kilómetro de aquellas que han sido cartografiadas por los vulcanólogos. Estas distorsiones observables entre puntos de vista pueden tener varias explicaciones. Por un lado, una parte importante de la población tiene un conocimiento limitado del fenómeno «lahar», como ya fue observado durante la sección referida a la percepción de los fenómenos volcánicos, por lo cual le es difícil imaginar la extensión posible de los mismos. Por otro lado, algunas personas consideran que otros productos, aparte de los lahares, como por ejemplo las cenizas, las bombas volcánicas o la lava volcánica, podrían constituir fuentes

13 A partir del conjunto de la muestra se constituyeron estratos que han permitido realizar mapas de percepción de los espacios más expuestos según el nivel de educación, la edad o la localización de las personas interrogadas (ver Salazar, 2008). 
de peligro importantes, a pesar de la distancia. Por último, resulta que los mapas de amenazas volcánicas del Instituto Geofísico, a veces publicados por la prensa o presentados en talleres, seminarios o campañas de sensibilización por parte de las autoridades locales, han influido sobre ciertas personas: particularmente el mapa de 1988, que representaba zonas expuestas más extensas que las del mapa actualizado de 200414. Sin embargo, se puede observar una excepción en el sector de «Playa Chica», al pie del cerro Ilaló y al noreste del «El Triángulo». En este caso, la zona altamente expuesta de 2004 es más extensa que la de 1988 e incluye al sector de Playa Chica. La población muestra otro punto de vista, cuya transcripción en el espacio es muy cercana a la del zonaje de 1988.

\section{PERCEPCIÓN DE LOS RIESGOS ASOCIADOS A UNA ERUPCIÓN DEL COTOPAXI}

La percepción de los riesgos considera los efectos de las erupciones volcánicas en términos de consecuencias para las personas y los bienes. En la encuesta, la percepción del riesgo ha sido considerada de dos maneras: por un lado, el punto de vista de los interrogados sobre los daños potenciales a escala del Valle de Los Chillos y, por otro lado, la percepción del riesgo personal; en otras palabras, la percepción del riesgo de las personas encuestadas en su lugar de residencia.

\section{1. Percepción del riesgo a escala del Valle de Los Chillos}

La gran mayoría de los interrogados (92\%) estima que las consecuencias de una erupción serían desastrosas para el Valle de Los Chillos. Este punto de vista encaja con aquel mostrado en lo referido a la magnitud probable de la erupción, donde $68 \%$ de las personas contemplan una erupción de gran tamaño y $11 \%$ una de tamaño mediano. Las consecuencias percibidas son poco diferenciadas, puesto que estas atañen en las mismas proporciones las pérdidas humanas, los daños materiales y las actividades económicas. Se citan, pero en escasa proporción, otras consecuencias, tales como la afectación de las tierras cultivables y del ganado o la paralización de los medios de transporte. Finalmente, muy pocas personas (10 \%) consideran una erupción sin pérdida de vidas humanas, lo que hace presagiar la gran inquietud que habrá que manejar el día en que el volcán presente signos anunciadores más claros que en 2002.

\section{2. Percepción del riesgo en el lugar de residencia}

El riesgo considerado corresponde aquí a lo que piensan las personas sobre los riesgos que corren en su lugar de residencia. Como lo indica el cuadro 2,

${ }^{14}$ El artículo de J. Robert, R. D’Ercole, P. Pigeon y T. Serrano (pp. 709-733) permite comprender la razón de las diferencias entre los mapas de 1988 y 2004. 
278 personas (o sea más de 45 \% de la muestra) estiman que serían personal y directamente muy afectadas en caso de erupción del Cotopaxi en términos de daños materiales (75 \% de esta población) y de daños a las actividades (44\%). Un $40 \%$ estima que su vida estaría en peligro. Para $27 \%$ de la muestra, los daños serían menores, limitándose a pérdidas materiales y a diversas perturbaciones del funcionamiento territorial. Solamente 13,5 \% de los interrogados estima que una erupción no los inquietaría y un $14 \%$ no se pronuncia.

Cuadro 2 - Grado de percepción del riesgo de los encuestados en su lugar de residencia

\begin{tabular}{|l|l|r|r|}
\hline $\begin{array}{c}\text { Grado de } \\
\text { afectación } \\
\text { esperado* }\end{array}$ & Grado de percepción del riesgo & Frecuencia & Porcentaje \\
\hline Mucho & Alto a muy alto & 278 & 45,2 \\
\hline Poco / Sí** & Relativamente alto & 168 & 27,3 \\
\hline No & Bajo a muy bajo & 83 & 13,5 \\
\hline No sabe & Relativamente bajo & 86 & 14,0 \\
\hline & Total & $\mathbf{6 1 5}$ & $\mathbf{1 0 0}$ \\
\hline
\end{tabular}

Fuente: Salazar, 2008

* Respuesta a la siguiente pregunta: «CCómo considera Ud. que su sitio de vivienda podría verse afectado en caso de una erupción?»

** Respuesta que se presentó en aquellas personas (9) que pensaban estar amenazadas en su lugar de residencia, pero que no pudieron evaluar el grado de afectación.

La figura 8, construida a partir de estos datos, representa la proporción, por unidades espaciales, de los entrevistados que tienen un nivel de percepción del riesgo en su lugar de residencia de alto a muy alto. Como en el mapa de percepción de los espacios más expuestos al Cotopaxi, este también presenta diferencias sensibles con el zonaje de los vulcanólogos. Las personas que habitan en las zonas establecidas por los vulcanólogos como de alto riesgo, se sienten amenazadas en grados variables: muy fuertemente en algunos casos (por ejemplo, las zonas de San Rafael, ESPE o Yaguachi), y mucho menos en otras áreas como San Gabriel, Mirasierra o Playa Chica. Encontramos, por lo menos en parte, los efectos del mapa de amenazas del Instituto Geofísico, pues la última versión de 2004 aparentemente no es conocida por una gran parte de la población. El grado de percepción del riesgo es muy elevado en las personas localizadas entre los brazos de los ríos Pita y Santa Clara, aun cuando están situadas fuera de las zonas de riesgo de lahares de los vulcanólogos. La población residente en esta zona, como toda la población interrogada (fig. 7), coincide en este punto, lo que evidencia un sector donde el manejo de emergencia será particularmente complejo15.

15 Es posible que se produzca, por ejemplo, una evacuación espontánea de una gran parte de la población residente, a pesar de que las autoridades estiman que la zona puede servir de albergue, ya que según los vulcanólogos esta estaría fuera del alcance de los lahares. 


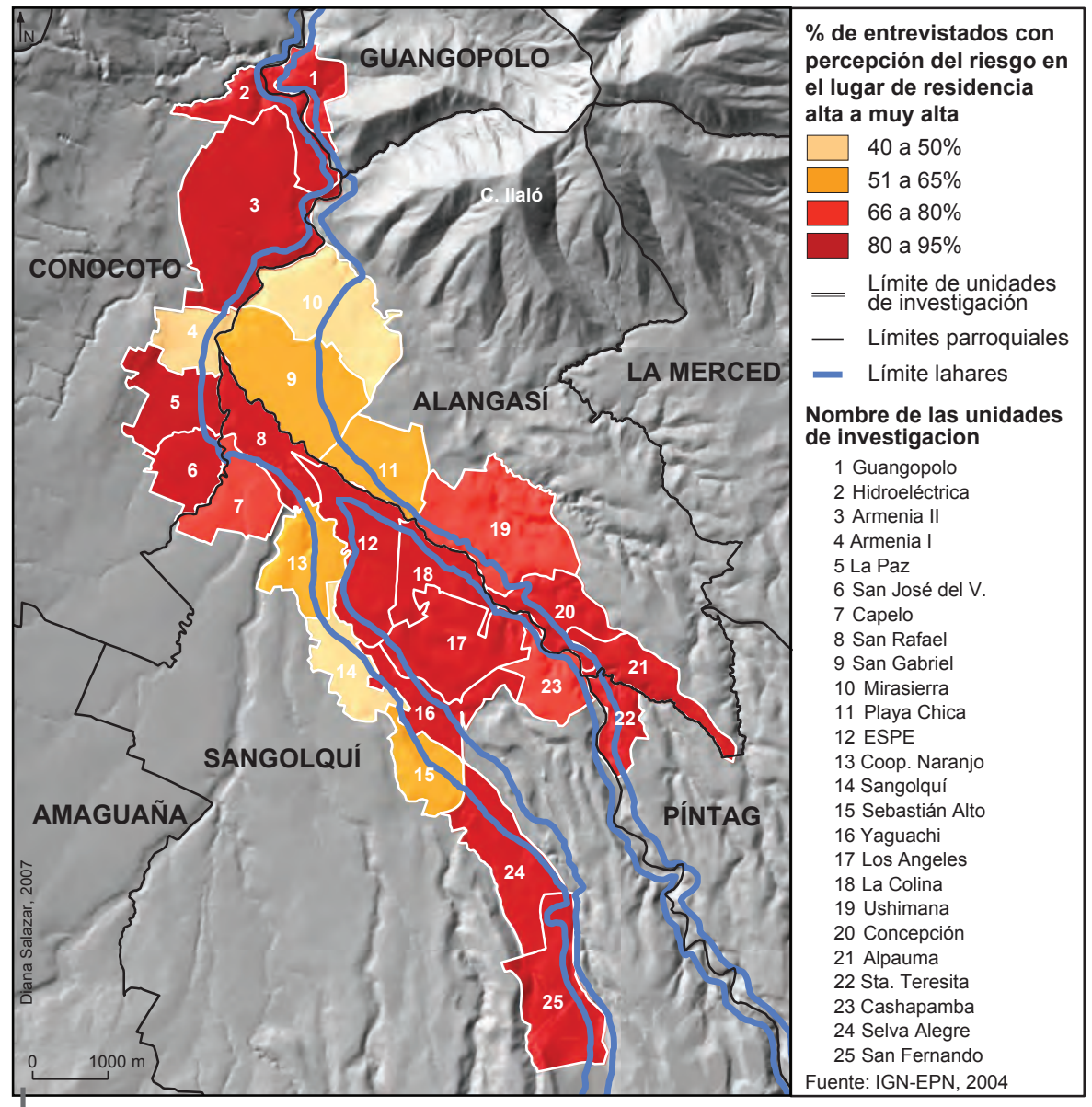

Figura 8 - Grado de percepción del riesgo de los encuestados en el lugar de residencia, según unidades de investigación

Fuente: D. Salazar, 2008

Por último, las zonas consideradas de riesgo por los residentes van más allá de las identificadas por los vulcanólogos, en particular en el norte (Conocoto y Guangopolo) y al este, al sur de la parroquia de Alangasi. Es interesante observar que el espacio comprendido entre Alangasí y Píntag es considerado como altamente peligroso por sus habitantes, mientras toda la población interrogada no le presta sino una atención limitada (fig. 7). La situación se invierte en lo que concierne a Sangolquí: todas las personas interrogadas estiman que este espacio está muy expuesto, pero la población residente, en su conjunto, se considera en un lugar seguro, yendo más bien en el sentido del zonaje de los vulcanólogos. 


\section{CONCLUSIONES}

\section{Desde el conocimiento de la percepción del riesgo hacia la reducción de la vulnerabilidad}

Un manejo de crisis eficaz supone una adecuación lo más correcta posible entre los puntos de vista de los diferentes actores involucrados. Ahora bien, el ejemplo del Cotopaxi y del Valle de Los Chillos, a semejanza de las investigaciones anteriores (Montaña Pelée, Pichincha), pone en relieve la diversidad (e incluso la divergencia) de puntos de vista entre científicos y población, y también en el seno mismo de la población, en materia de percepción de las amenazas o de los riesgos.

En su conjunto, ya sea en términos de fuerza de la erupción como de extensión de las zonas afectadas, la mayoría de la población interrogada contempla una erupción colosal del Cotopaxi. Las cenizas, la lava, las piedras y bloques volcánicos, así como los lahares, aunque en menor proporción, son percibidos como los peligros más importantes. Además, una alta proporción de personas considera que esta erupción puede ocurrir muy rápidamente después del despertar del volcán o en cualquier momento.

Este punto de vista es sensiblemente diferente del de los vulcanólogos, que es más matizado. Estos últimos consideran que son posibles varios tipos de crisis volcánicas extendidas en el tiempo, con fases de actividades volcánicas previas a una gran erupción probablemente largas (mínimo varias semanas). Estas crisis se caracterizan por una gran variedad de fenómenos volcánicos (que excluyen, sin embargo, los flujos de lava fuera del edificio volcánico) que tienen un potencial destructor y un impacto espacial variables. El desarrollo en el tiempo de las crisis volcánicas no puede ser previsto, según un sistema de probabilidades, solo cuando el volcán se ha reactivado y de acuerdo a las características de esta actividad. Por consiguiente, existen muchas incertidumbres por parte de los científicos y esto tiende a contrastar con algunas certezas por parte de la población expuesta.

Sobre este punto, pero también sobre otros (espacios expuestos, por ejemplo), el contraste entre los saberes científicos y de la población es menos fuerte hoy que hace 20 años, como lo indica la comparación con los estudios de R. D’Ercole. La población ha madurado: está mejor informada y sensibilizada por las numerosas erupciones que ha vivido Ecuador desde 1998. El grado de percepción del riesgo asociado al Cotopaxi se ha vuelto alto en su conjunto, pese a que a fines de los años 1980 era considerado muy débil. Las zonas de riesgo parecen ser mejor conocidas, aunque los fenómenos volcánicos son todavía mal apreciados, y las incertidumbres son más aceptadas.

La información que ofrece este tipo de investigación presenta indiscutiblemente un interés para los gestores de los riesgos y de las crisis, pero cabe plantearse la pregunta acerca de su utilidad real, en términos de acción preventiva y de reducción de la vulnerabilidad de la población. Frecuentemente se mencionan dos limitaciones. Por un lado, el carácter empírico de la investigación. Como lo señala Mary Douglas, no existe teoría para comprender la percepción humana 
del riesgo, lo que tiende a desembocar sobre investigaciones empíricas (Douglas, 1987). Pero esto no es un verdadero freno para la acción.

Más problemática es la segunda crítica. Según la misma autora, estas investigaciones presentan el defecto de enfatizar las razones por las cuales la racionalidad de los individuos no ha sido puesta en práctica y, «cuando los investigadores encuentran pruebas de un razonamiento irracional, recomiendan una mejor educación del público, para paliar las supuestas fallas de su razonamiento» (Douglas, 1987: 57).

Los estudios realizados en el Valle de Los Chillos indican claramente que uno no se puede contentar con una visión tan tajante separando a los que saben de aquellos que supuestamente no saben y tratando de aumentar al máximo el primer grupo mediante campañas de educación. Es cierto que las campañas de educación basadas en estudios como los nuestros pueden reducir algunas vulnerabilidades, mejorando por ejemplo el conocimiento acerca de los fenómenos volcánicos, pero estas no pueden resolver todos los problemas. En efecto, existen muchas incertidumbres cuando se habla de riesgo volcánico (fenómenos, fuerza, extensión espacial, desarrollo en el tiempo, etc.) y los científicos son los primeros en reconocerlo. Enseñar a la población los límites geográficos de las zonas expuestas a los lahares, establecidas por los vulcanólogos, no garantiza la calidad del manejo de una crisis. El saber de los científicos es impreciso, al igual que el de la población, cada uno con sus propios criterios. Por esta razón, los mapas de percepción de los espacios expuestos o de percepción de los riesgos en el lugar de residencia, como los que hemos construido, son tan o más útiles en términos de manejo de crisis o de preparación para el manejo de crisis, que los mapas de amenazas. Son mapas que destacan los lugares donde se plantearán problemas, incluso si no son espacios peligrosos desde el punto de vista científico. El caso del sector comprendido entre los ríos Pita y Santa Clara es uno de los más ilustrativos. A partir del mapa de amenazas de los científicos, la población de esta zona está segura, aun cuando pueda estar aislada en caso de erupción. Pero el mapa de percepción del riesgo de la población que vive en este sector muestra que esta se considera no solo entrampada, sino también directamente amenazada. La diferencia es importante y tomar en cuenta el punto de vista de la población, gran parte de la cual querrá ser evacuada el día en el que el volcán amenace, parece necesario para mejorar la prevención, el sistema de manejo de crisis y, en consecuencia, para reducir la vulnerabilidad de la misma población. Los estudios de percepción de riesgos se vuelven entonces muy útiles si el propósito no es el de conseguir la adhesión de un máximo de población a un punto de vista único, considerado objetivo, sino el de unir de manera constructiva las diferentes perspectivas. 


\section{Referencias citadas}

ALEXANDER, D., 2000 - Confronting catastrophe, 282 pp.; Hertfordshire, Inglaterra: Terra Publishing.

BAILLY, A., 1987 - Des images mentales de Fribourg; trois images pour deux groupes culturels. Cahiers de I'Institut de Géographie de Fribourg, 5: 99-106.

BAILLY, A., 1992 - Les représentations en géographie. In: Encyclopédie de Géographie (A. Bailly, R. Ferras \& D. Pumain, eds.): 371-383; París: Economica.

BAILLY, A., BEAUMONT, C., HURIOT, J.-M. \& SALLEZ, A., 1995 - Représenter la ville, 112 pp.; París: Economica. Coll. « GÉO Poche».

CLAVAL, P., 1992 - Géographie et sociologie. In: Encyclopédie de Géographie (A. Bailly, R. Ferras \& D. Pumain, eds.): 75-91; París: Economica.

DAUPHINÉ, A., 2001 - Risques et catastrophes. Observer, spatialiser, comprendre, gérer, 288 pp.; París: Armand Colin.

DAVIS, M. \& RICCI, T., 2004 - Perceptions of Risk for Volcanic Hazards in Italy: A Research Note. Internacional Journal of Sociology and Social Policy, vol. 24, n. ${ }^{\mathbf{1 0}} \mathbf{1 1}$ : 159-165.

D'ERCOLE, R., 1991 - Vulnérabilité des populations face au risque volcanique. Le cas de la région du volcan Cotopaxi (Équateur) - Vulnerabilidad poblacional frente al riesgo volcánico. El caso de la región del volcán Cotopaxi (Ecuador), 460 pp.; Grenoble: Université Joseph Fourier. Thèse de doctorat.

D'ERCOLE, R., 2008 - Percepción del riesgo y vulnerabilidad. Aplicación al riesgo volcánico. In: La geofísica y su aporte en la reducción de riesgos de desastres naturales (Hernando Tavera, editor): 121-124; Lima: Ediciones Nova Print SAC. Simposio internacional «Terremotos, volcanes, tsunamis, deslizamientos, sismicidad inducida y efectos asociados», Lima 12-14 de agosto 2008.

D'ERCOLE, R. \& RANÇON, J.-P., 1994 - La future éruption de la montagne Pelée: Risque et représentations. Mappemonde, $\mathbf{n} .^{\circ}$ 4: 31-36.

D'ERCOLE, R. \& RANÇON, J.-P., 1999 - Représentation des risques liés à une éruption de la montagne Pelée, Martinique. Confrontation des points de vue des populations proches (Saint-Pierre, Le Prêcheur et Le Morne-Rouge) et des volcanologues. In: Les Antilles, Terres à risques: 165-182; París: Editions KARTHALA, GEODE Caraïbe.

D'ERCOLE, R. \& METZGER, P., 2002 - Diferenciaciones espaciales y sociales, representaciones y manejo del riesgo volcánico en Quito. In: Gestión de riesgos y prevención de desastres: 40-54; Quito, Ecuador: Flacso, Coopi, ECHO.

DOMINEY-HOWES, D. \& MINOS-MINOPOULOS, D., 2004 - Perceptions of hazard and risk on Santorini. Journal of Volcanology and Geothermal Research, 137: 285- 310.

DOUGLAS, M., 1970 - Natural symbols: explorations in Cosmology, 178 pp.; Nueva York: Pantheon Books.

DOUGLAS, M., 1987 - Les études de perception du risque : un état de l'art. In: La société vulnérable. Évaluer et maîtriser les risques (J.-L. Fabiani \& J. Theys): 55-60; París: Presses de l'École Normale Supérieure.

DOUGLAS, M. \& WILDAVSKY, A. B., 1982 - Risk and culture: An essay on the selection of technical and environmental dangers, 224 pp.; Berkeley: University of California Press.

GOULD, P., 1966 - On mental maps. University of Michigan, Department of Geography, discussion paper $\mathrm{n} .^{\circ} 9$. 
GOULD, P. \& WHITE, R., 1974 - Mental maps, 204 pp.; Penguin: Harmondsworth.

LAVIGNE, F., DE COSTER, B., JUVIN, N., FLOHIC, F., GAILLARD, J.-C., TEXIER, P., MORIN, J. \& SARTOHADI, J., 2008 - People's behaviour in the face of volcanic hazards: Perspectives from Javanese communities, Indonesia. Journal of Volcanology and Geothermal Research, vol. 172, n⿳ 3-4: 273-287.

LYNCH, K., 1960 - The image of the city, 220 pp.; Cambridge, Massachusetts: The Mit Press.

PEAKE, S., 2004 - Analysis of spatial distortions in a mental map using GPS and GIS; New Zealand: University of Otago. Honours thesis (http://eprints.otago.ac.nz/385/).

PERETTI-WATEL, P., 2003 - Sociologie du risque, 286 pp.; París: Armand Colin. Collection U.

PERRY, R.W., LINDELL, M. K., GREENE, M. R., 1983 - Threat perception and public response to volcano threat. Journal of Social Psychology, 116: 199-204.

ROBERT, J., 2007 - Vulnérabilité de la population exposée aux lahars du volcan Cotopaxi dans le Valle de Los Chillos, Quito-Équateur, 97 pp.; Grenoble: Université de Savoie. Mémoire de Master II Recherche.

SALAZAR, D., 2008 - Percepción de amenazas y riesgos de la población del Valle de los Chillos (Ecuador) frente a una potencial erupción del volcán Cotopaxi, 187 pp.; Quito: Pontificia Universidad Católica del Ecuador. Disertación previa a la obtención del título en Ingeniería Geográfica y Desarrollo Sustentable con mención en Ordenamiento Territorial.

SLOVIC, P., 1987 - Perception of risk. Science, New Series, Vol. 236, n. ${ }^{\circ}$ 4799: 280-285.

VEYRET, Y., BEUCHER, S. \& REGHEZZA, M. (ed.), 2004 - Les risques, 206 pp.; Rosny-sousBois: Breal. Coll. Amphi Géographie.

ZAYED, J., 2006 - Analyse des risques sur la santé humaine : perception des risques, principe de précaution. Ponencia presentada en el coloquio international « Développement, environnement et santé », Bamako, Mali (12-16 juin 2006). 